\title{
Cutaneous collagenous vasculopathy: light and transmission electron microscopy*
}

\author{
Debora Sarzi Sartori ${ }^{1,2}$, Hiram Larangeira de Almeida Jr., ${ }^{2,3}$, Timotio Volnei Dorn ${ }^{4}$, Caroline Pires Ruas ${ }^{5}$
}

DOI: http:/ /dx.doi.org/10.1590/abd1806-4841.20198166

\begin{abstract}
Cutaneous collagenous vasculopathy is a rare acquired idiopathic microangiopathy characterized by progressive development of diffuse asymptomatic telangiectasias and histologically by accumulation of collagen type IV around the affected vessels. It is diagnosed by its clinical history, confirmed by light microscopy with collagen-specific immunostaining. We report a case of a patient with extensive acquired telangiectasias on the left arm, clinically resembling unilateral nevoid telangiectasia. Dilated blood vessels with thickened walls were observed in the dermis. Immunohistochemistry with collagen IV antibodies revealed marked collagen deposition around the vessels, confirming the diagnosis. Transmission electron microscopy observed duplicate and triplicate vascular basal membrane associated with deposition of amorphous material around the membranes.
\end{abstract}

Keywords: Collagen type IV; Microscopy, electron, transmission; Skin diseases, vascular; Vascular diseases

\section{INTRODUCTION}

Cutaneous collagenous vasculopathy $(\mathrm{CCV})$ is a rare acquired idiopathic microangiopathy, first described in 2000. It is characterized by the progressive development of diffuse asymptomatic telangiectasias over the skin and by the accumulation of collagen type IV in the outer vessel walls. It is probably an underdiagnosed condition. We describe a 51-year-old patient with skin lesions in the left forearm and arm with typical histological (light and electron microscopy) and immunohistochemical findings.

\section{CASE REPORT}

A 51-year-old woman presented with asymptomatic telangiectatic macules for 20 years. The lesions appeared after the first pregnancy, initially on the distal portion of the left forearm and progressed to the medial portion of the left arm (Figure 1A and 1B). Dermoscopy showed clinical vascular features of the lesion (Figure 1C). The patient denied bleeding, conjunctival telangectasias or family history of similar cases. Additional tests including viral serologies (HIV, B and C hepatitis), C-reactive protein, erythrocyte sedimentation rate, and antinuclear antibodies were normal. Patient was diagnosed with mammary ductal carcinoma 2 years ago and treated with left quadrantectomy and postoperative radiotherapy. She is undergoing cancer follow-up, with no signs of relapse. Patient denied other comorbidities or change in her condition during oncologic treatment.

Light microscopy with HE staining revealed dilated superficial and medial dermal blood vessels (Figure 2A and 2B). Periodic acid-Schiff (PAS) staining showed thickening of the blood vessel walls (Figure 2C and D). Immunohistochemical staining for collagen type IV revealed extensive deposition of type IV collagen around the blood vessels (Figure 3A). On higher magnification, increased vessel wall thickening and increased tortuosity were observed (Figure 3B); these findings were not seen in the skin of normal controls (Figure 3C).

Transmission electron microscopy of the dermis showed vessels with duplicate and triplicate basal membrane in the affected area, as well as marked amorphous material deposition among and around the membranes (Figure 4).

\footnotetext{
Received 07 February 2018.

Accepted 27 May 2018. (RS), Brazil; Electron Microscopy Center of the South, Fundação Universidade de Rio Grande, Rio Grande (RS), Brazil.

Financial support: None.

Conflict of interest: None.

Academic Hospital, Universidade Federal de Pelotas, Pelotas (RS), Brazil.

Post-Graduation Program in Health, Universidade Católica de Pelotas, Pelotas (RS), Brazil.

Department of Dermatology, Universidade Federal de Pelotas, Pelotas (RS), Brazil.

Dermacenter Alto Vale, Rio do Sul (SC), Brazil.

Electron Microscopy Center of the South, Fundação Universidade de Rio Grande, Rio Grande (RS), Brazil.
}

* Work conducted at the Post-Graduation Program in Health, Universidade Católica de Pelotas, Pelotas (RS), Brazil; Universidade Federal de Pelotas, Pelotas

MAILING AdDRESS:

Hiram Larangeira de Almeida Jr

E-mail: hiramalmeidajr@hotmail.com 


\section{DISCUSSION}

$\mathrm{CCV}$ is a primary form of acquired microangiopathy that resembles the superficial telangiectasias of generalized essential telangiectasia, however, with distinct histological, immunohistochemical and electronic microscopy features. It was first described by Salama and Rosenthal in $2000 .^{1}$

According to the cases reported to date, it affects both sexes, mostly women, various age groups, although mainly the middle-aged and elderly. Most cases of CCV described have been associated with other concomitant diseases, most commonly hypertension and cardiovascular disease. ${ }^{1,2}$

Clinically, CCV presents as telangiectatic macules that typically begin on the lower extremities and then spread to the trunk and upper extremities and may become generalized in some cases. The lesions are usually asymptomatic, with occasional pruritus. There are no reports of mucosal or nail involvement or any bleeding events. Other primary vascular disorders in the differential diagno-
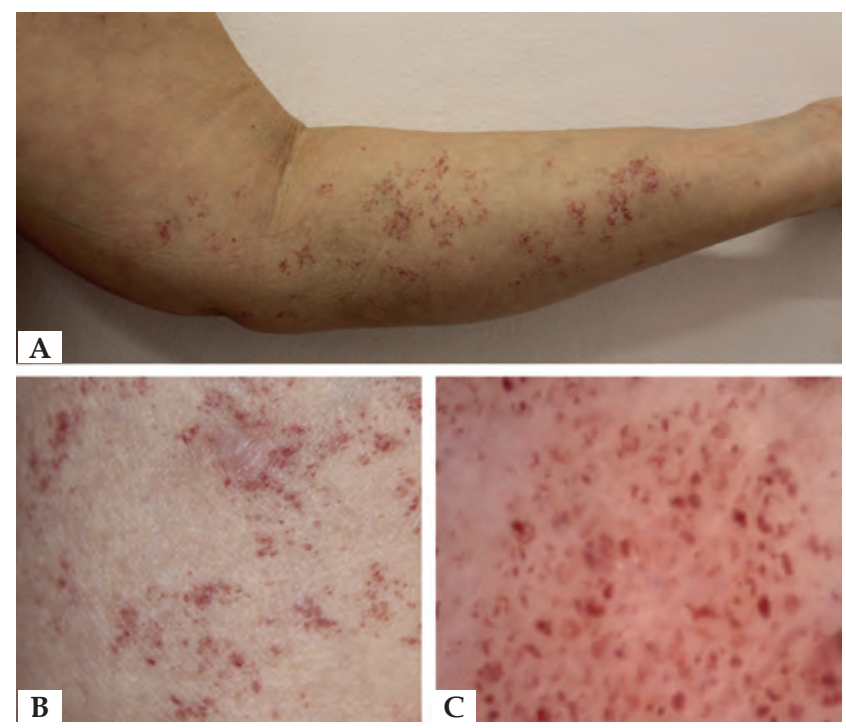

Figure 1: A - Clinical aspect on the left arm. B - Detail of telangiectatic lesions. C - Dermoscopy showing the vascular nature of the lesions
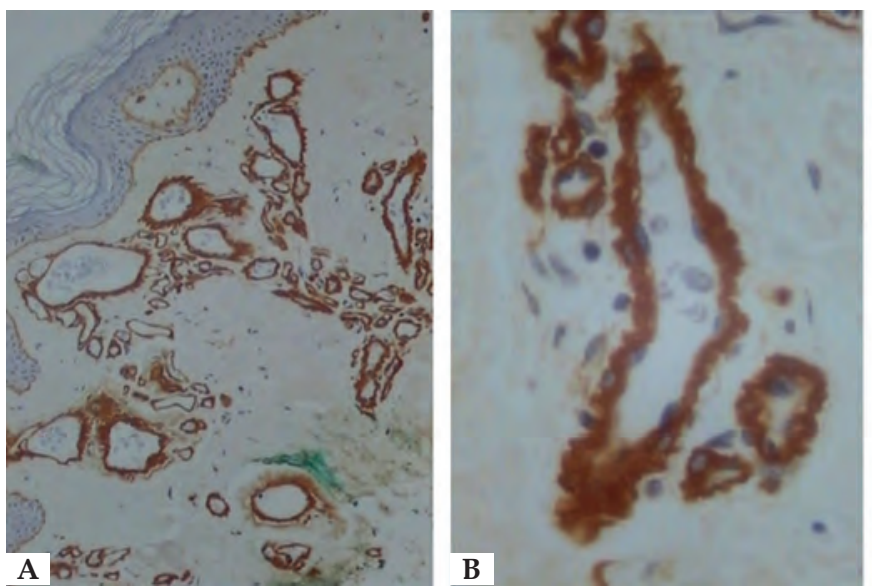

sis of CCV include generalized essential telangiectasia, hereditary hemorrhagic telangiectasia, hereditary benign telangiectasia and ataxia-telangiectasia, and particularly with unilateral nevoid telangiectasia, the case here described has a segmental involvement; however, in the latter, the vessels have normal walls.,

The histological findings are characteristic and may differ from other vascular disorders. CCV has dilated vascular structures that contain deposits of eosinophilic hyaline material within the vessel walls, markedly stained with PAS, periodic acid-Schiff-diastase (PAS-D) and Masson's trichrome.

Immunohistochemical staining for type IV collagen is strongly positive. Ultrastructure findings revealed changes in post-capillary venules due the presence of characteristic reduplication of the basal lamina, as shown in this case. Some authors have reported the presence of collagen band deposition with altered thickening in the outer extremities of the affected vessel walls, although not pathognomonic. ${ }^{1,2,5}$
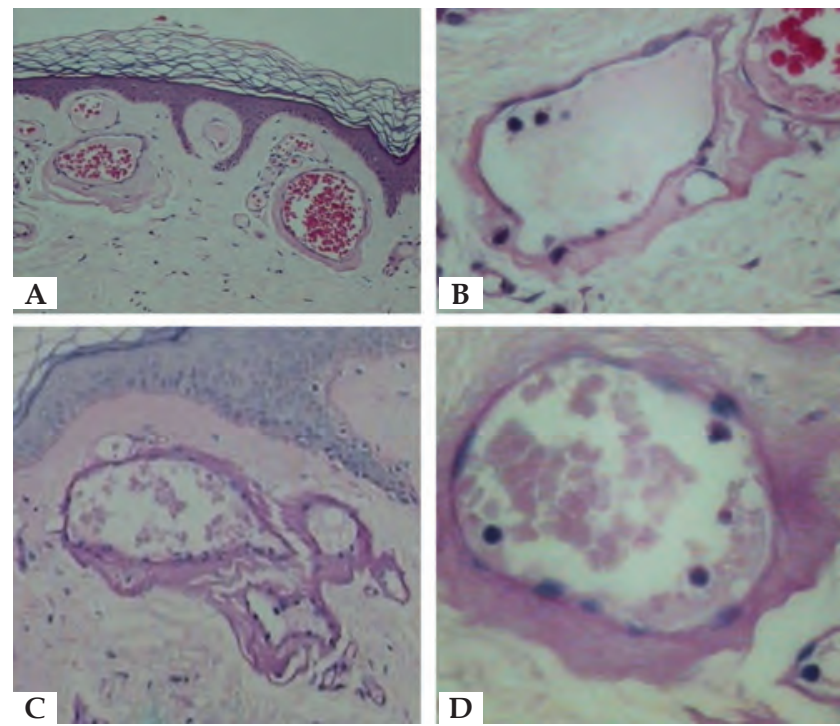

Figure 2: Light Microscopy - A - Ectatic superficial vessels (Hematoxylin and eosin x150). B - Detail of a vessel with thick wall (Hematoxylin and eosin $x 400)$. C - Three vessels with thick wall (Periodic acid-Schiff $\times 150$ ). D - Detail of a vessel with thick wall (Periodic acid-Schiff, $x 400$ )

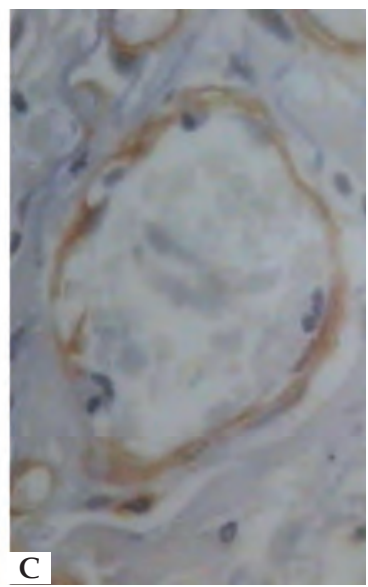

FIGURE 3: Immunohistochemistry with anti-collagen IV antibody. A - Demarcation of the wall of ectatic dermal vessels (x150). B - Detail of the clear demarcation of tortuous vessels (x400). C - Normal control with a normal vessel wall $(x 400)$ 

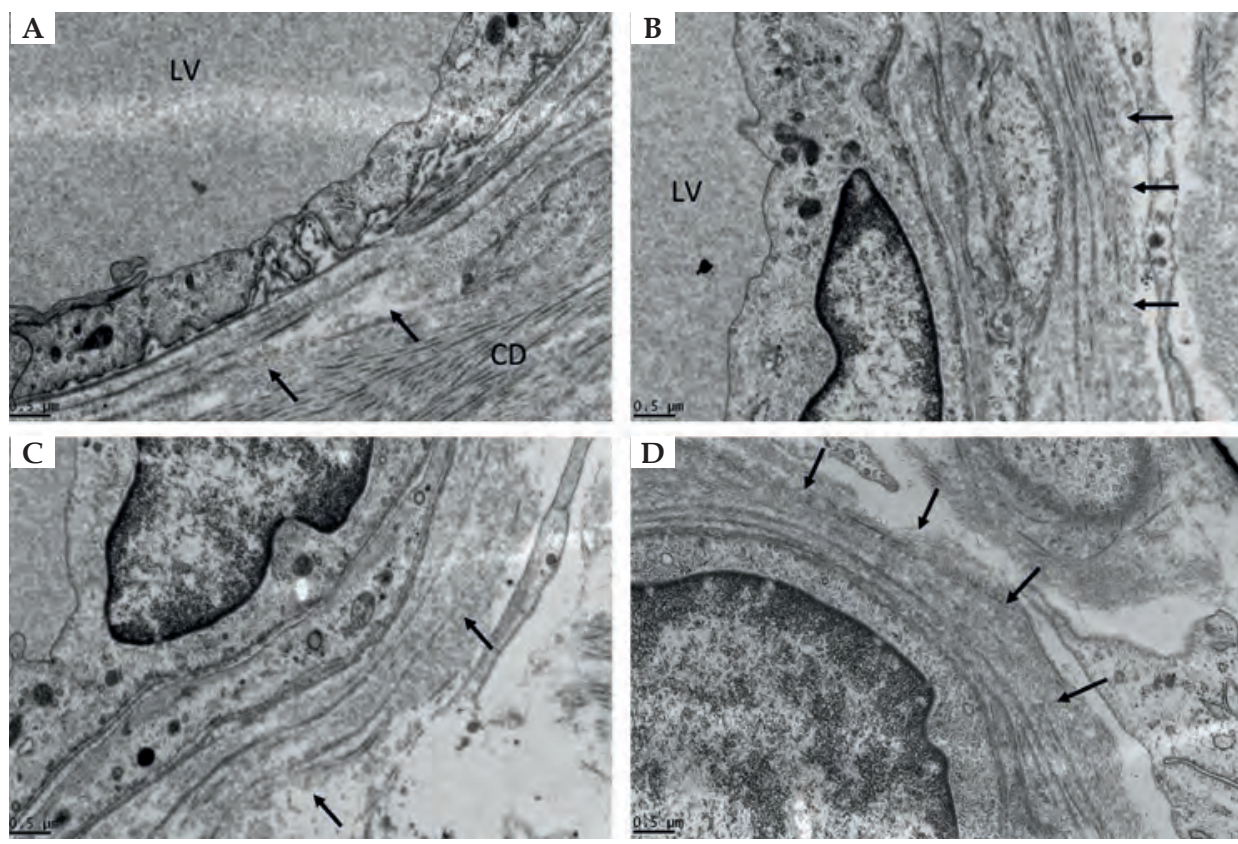

Figure 4: Transmission electron microscopy

A - Duplicated basement membrane (arrows); LV - vascular lumen; CD - dermal collagen $(x 25,000)$. B,C e D - Tripled basement membrane (arrows) and deposition of amorphous material $(x 25,000)$

Its aetiology is still unknown, but it is believed to be related to a genetic defect in collagen synthesis, systemic diseases, and to the use of medications. Salama et al., ${ }^{5}$ and others authors, ${ }^{6,7}$ suggest that the repeated damage to the endothelial cells can induce the formation of intravascular occlusive microthrombi, leading to endothelial hyperplasia and perivascular fibrosis, which were reported based on findings of intravascular occlusive microthrombi observed in the histology of the CCV lesions. Our patient had no comorbidities.

A few therapeutic options have been described in literature. Some authors reported cases of effective treatment with the use of laser systems (595-nm pulsed dye laser and $1064 \mathrm{~nm} \mathrm{Nd:YAG).}{ }^{8}$

Our results are consistent with the findings shown by other authors, found in the literature. $\square$

\section{REFERENCES}

1. Salama S, Rosenthal D. Cutaneous collagenous vasculopathy with generalized telangiectasia: an immunohistochemical and ultrastructural study. J Cutan Pathol. 2000;27:40-8.

2. Salama SS. Cutaneous Collagenous Vasculopathy: A New Case Series With Clinicopathologic and Ultrastructural Correlation, Literature Review, and Insight Into the Pathogenesis. Am J Dermatopathol. 2015;37:368-75.

3. Wenson SF, Jan F, Sepehr A. Unilateral nevoid telangiectasia syndrome: A case report and review of the literature. Dermatol Online J. 2011:17:2.

4. Smith JA, Kamangar F, Prakash N, Fung MA, Konia T, Fazel N. Unilateral nevoid telangiectasia syndrome (UNTS) associated with chronic Hepatitis C virus and positive immunoreactivity for VEGF. Dermatol Online J. 2014;20. pii: 13030/ qt8g9268sf.
5. Salama S, Chorneyko K, Belovic B. Cutaneous collagenous vasculopathy associated with intravascular occlusive fibrin thrombi. J Cutan Pathol. 2014;41:386-93.

6. Kanitakis J, Faisant M, Wagschal D, Haftek M, Claudy A. Cutaneous collagenous vasculopathy: ultrastructural and immunohistochemical study of a new case. Am J Clin Dermatol. 2010;11:63-6.

7. Basso D, Ribero S, Blazek C, Dietrich N, Beltraminelli $\mathrm{H}$, Ramelet AA, et al. Cutaneous Collagenous Vasculopathy: A Rare Form of Microangiopathy Successfully Treated with a Combination of Multiplex Laser and Optimized Pulsed Light with a Review of the Literature. Dermatology. 2016;232:107-11.

8. Echeverría B, Sanmartín 0, Botella-Estrada R, Vitiello M. Cutaneous collagenous vasculopathy successfully treated with pulsed dye laser. Int J Dermatol. 2012:51:1359-62.

Approval of the final version of the manuscript; Elaboration and writing of the manuscript; Obtaining, analyzing and interpreting the data; Effective participation in research orientation; Critical review of the literature; Critical review of the manuscript

Hiram Larangeira de Almeida Jr. $\quad$ (D) ORCID 0000-0003-0705-1778

Approval of the final version of the manuscript; Conception and planning of the study; Elaboration and writing of the manuscript; Obtaining, analyzing and interpreting the data; Effective participation in research orientation; Critical review of the literature; Critical review of the manuscript

Timotio Volnei Dorn $\quad$ (iD) ORCID 0000-0001-9666-6146

Approval of the final version of the manuscript; Conception and planning of the study; Elaboration and writing of the manuscript; Obtaining, analyzing and interpreting the data; Effective participation in research orientation; Critical review of the literature; Critical review of the manuscript

Caroline Pires Ruas $\quad$ (iD) ORCID 0000-0002-6917-1727

Approval of the final version of the manuscript; Conception and planning of the study; Elaboration and writing of the manuscript; Obtaining, analyzing and interpreting the data; Critical review of the literature; Critical review of the manuscript
}

How to cite this article: Sartori DS, Almeida Jr HL, Dorn TV, Ruas CP. Cutaneous collagenous vasculopathy: light and transmission electron microscopy. An Bras Dermatol. 2019;94(2):211-3. 